\title{
Percutaneous liver biopsy: another option of hepatic sample collection approach in single-cell sequencing studies?
}

\author{
Zi-Yuan Zou ${ }^{1,2}$, Jian-Gao Fan ${ }^{1,3}$ \\ ${ }^{1}$ Center for Fatty Liver, Department of Gastroenterology, Xinhua Hospital Affiliated to Shanghai Jiao Tong University School of Medicine, \\ Shanghai, China; ${ }^{2}$ Nanfang Hospital, Southern Medical University, Guangzhou, China; ${ }^{3}$ Shanghai Key Lab of Pediatric Gastroenterology and \\ Nutrition, Shanghai, China \\ Correspondence to: Jian-Gao Fan, MD. Center for Fatty Liver, Department of Gastroenterology, Xinhua Hospital Affiliated to Shanghai Jiao Tong \\ University School of Medicine, Shanghai Key Lab of Pediatric Gastroenterology and Nutrition, 1665 Kongjiang Road, Shanghai, China. Email: \\ fanjiangao@xinhuamed.com.cn. \\ Provenance and Peer Review: This article was a free submission to the editorial office, Annals of Translational Medicine. The article was sent for external \\ peer review.
}

Submitted Jan 11, 2020. Accepted for publication Mar 20, 2020.

doi: 10.21037/atm.2020.04.23

View this article at: http://dx.doi.org/10.21037/atm.2020.04.23

In this era of precision medicine, single-cell RNA sequencing (scRNA-seq), with the advantage of developing personalized phenotyping strategies that cross subcellular, cellular, and tissue scales to link our genome to disease pathogenesis, has been widely used in the transcriptomic landscape of various human organs, such as kidney (1), brain $(2)$, liver $(3,4)$ and so on, but the sample collection plan for scRNA-seq in human liver disease is still under heated debate. Writing in Nature, Ramachandran et al. (5) firstly used scRNA-seq to uncover newlydefined scar-associated TREM2+CD9+ macrophage and ACKR1+PLVAP+ endothelial cell subpopulations in the cirrhotic liver tissue obtained from liver transplantation, and dissected a complex, pro-fibrotic interactome between these cells and the collagen producing myofibroblasts. These intra-scar pathways represent a rational approach to the discovery of novel anti-fibrotic treatments for patients with chronic liver disease.

In this first study exploring non-hepatocellular human liver disease by scRNA-seq, the action of obtaining nonischemic cirrhotic liver tissue from patients undergoing orthotopic liver transplantation has been validated, with safety and ethics. Notably, using this sample collection approach, Ramachandran and colleagues could obtain a wedge biopsy of sample (2-3 grams), which included largeenough cellular contents.

Since the majority of patients with cirrhosis waiting for liver transplantation are decompensated (6), it is inevitable that only end-stage liver disease (ESLD) will be included in this program, which implies that their dissection may suffer from bias. Findings in the past decade also suggest that decompensated cirrhosis is characterized by exuberant systemic inflammation, in which several vasoactive molecules and inflammatory cytokines, such as nitric oxide (NO) and tumor necrosis factor (TNF) alpha may contribute to changes in the endothelium $(7,8)$. Analogously, previous studies reported that plasma from patients with ESLD significantly changed the function of monocyte-derived macrophages in a prostaglandin E2 (PGE2) receptor-dependent manner, effects not seen in compensated cirrhosis (9). Therefore, it is difficult to rule out the possibility that decompensated status may alter the phenotype of macrophage and endothelial cell subpopulations in the fibrotic niche.

On another perspective, the main outcome monitored in Ramachandran and colleagues' study was the branching differentiation trajectory from peripheral blood monocytes into scar-associated macrophages, whilst the authors did not successfully trace the origin of scar-associated endothelial cells. In this study, trajectory analysis showed poor pseudotemporal dynamics between scar-associated endothelial cells and any other phenotype of hepatic endothelial cells. The reason may be the absence of multiple stages of hepatic fibrosis, such as significant fibrosis. Another scRNA-seq study uncovered the gene expression of non-parenchymal cells (NPCs; including endothelial 


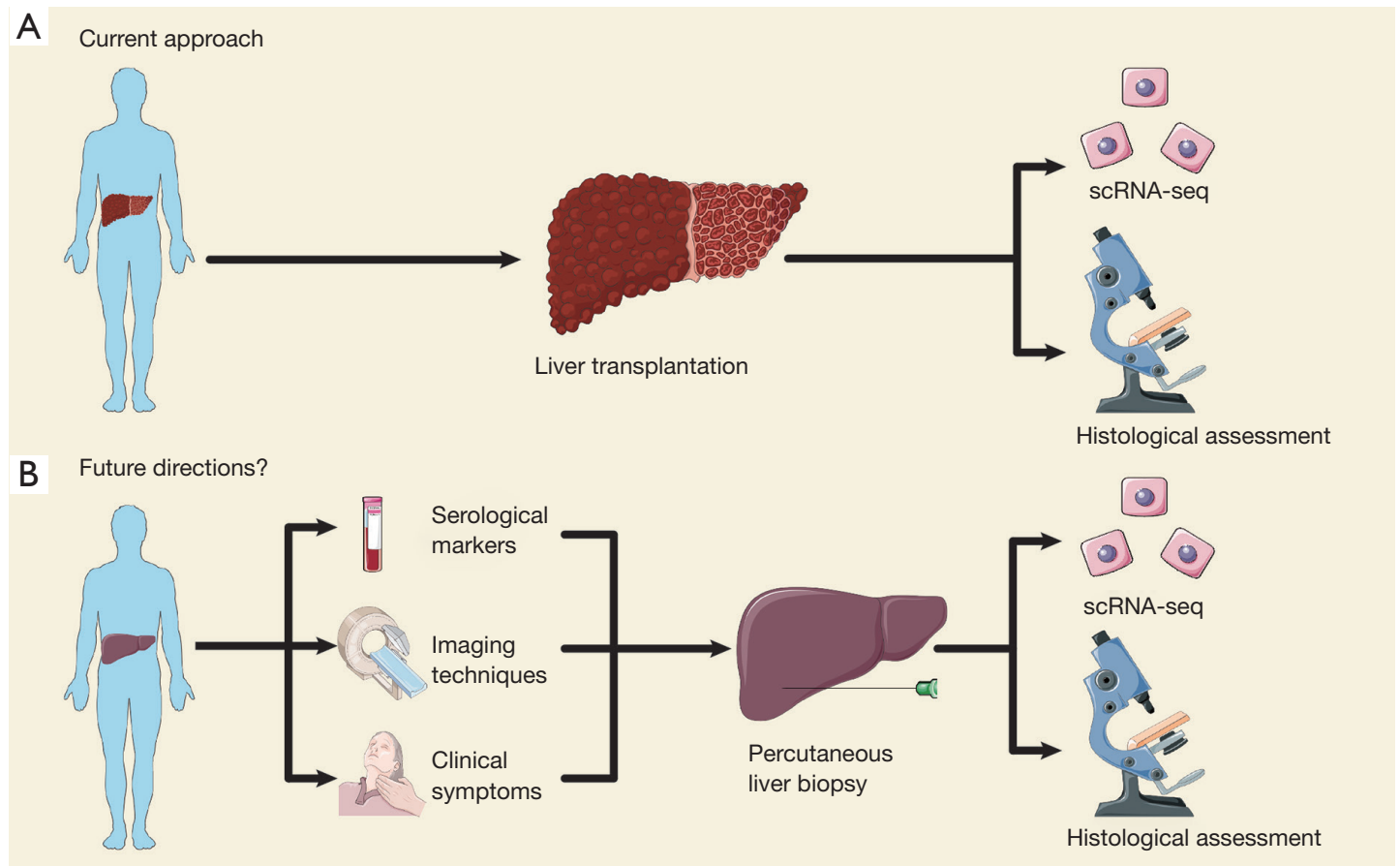

Figure 1 Sample collection approach for single-cell RNA sequencing (scRNA-seq) in human liver disease. Ramachandran et al. (5) uncover newly-defined scar-associated macrophage and endothelial cell subpopulations in the cirrhotic liver tissue obtained from liver transplantation. (A) In this study, liver tissue was obtained from patients undergoing orthotopic liver transplantation. However, the majority of patients with cirrhosis waiting for liver transplantation are decompensated. Since decompensated status and its concomitant systemic inflammation may alter the phenotype of macrophage and endothelial cell, it is difficult to rule out the bias from this sample collection process. (B) Percutaneous liver biopsy could perhaps lessen the bias. Such an approach might be used for both decompensated and compensated chronic liver disease. Specifically, the indications of biopsy need to be identified by serological markers, imaging techniques and clinical symptoms. All patients should provide informed consent to ensure that the approach is ethical. After percutaneous liver biopsy, histological assessment was performed as the gold standard for pathological diagnosis and the remaining tissue would be then transported to the laboratory for single-cell analyses and other concomitant assays (such as scRNA-seq, spatial transcriptomics and proteomics).

cells) in non-cirrhotic nonalcoholic steatohepatitis by using rodent models (10). Unfortunately, the unique markers of scar-associated endothelial cells, ACKR1 and PLVAP were not reported in this study. Considering the interspecific differences, comparatively continuous waves of gene expression identified by multistage biopsy of human liver might be required to dissect the founder cell of scarassociated endothelial cells.

Several unbiased sample collection approaches are open for future study. In this regard, we recommend that, after pathological examination for the primary purpose of diagnosis, the remaining liver biopsy specimens can be used for scRNA-seq. The idea behind percutaneous needle biopsy for procurement of clinical hepatic samples comes from highquality scRNA-seq research in the field of kidney disease (1).
According to the research published by Der et al. (1), tissue samples were obtained from patients undergoing clinically indicated renal biopsy and all patients provided informed consent concerning the use of renal samples for further research purposes. In terms of feasibility, the authors specified that the mean tissue mass of renal biopsy was $3 \mathrm{mg}$ (2-5 mg) and after filtering out abnormal cells from 21 renal tissue samples, 4,019 cells were included and sufficient for downstream bioinformatics analysis. With reference to Der and colleagues' study, we have designed a future direction and summarized the current approach (Figure 1).

Ramachandran and colleagues' study provided insight into a complex, orchestrated interplay between multiple nonparenchymal cell lineages including immunological, endothelial and mesenchymal cells. However, considering 
that hepatic samples from ESLD may lead to bias, further single-cell studies are recommended to validate the pathogenesis of hepatic fibrosis in liver biopsy samples. In addition, since some clinical trials were dual-biopsy to assess the efficacy of interventions $(11,12)$, the remaining hepatic samples could be used for scRNA-seq to elucidate pharmacological actions and mechanisms of drug resistance. With the emergence and development of early diagnostic techniques, interest in asymptomatic or compensated chronic liver disease has been gaining momentum. Thus, there is no doubt that percutaneous liver biopsy has great applicable potential in the mechanism research of human liver diseases at a single-cell level.

\section{Acknowledgments}

Funding: This study was supported by the National Key R\&D Program (2017YFC0908903), the National Natural Science Foundation of China (81873565), Shanghai Leading Talent Plan 2017, and Innovative Research Team of High-Level Local Universities in Shanghai.

\section{Footnote}

Conflicts of Interest: Both authors have completed the ICMJE uniform disclosure form (available at http://dx.doi. org/10.21037/atm.2020.04.23). The authors have no conflicts of interest to declare.

Ethical Statement: The authors are accountable for all aspects of the work in ensuring that questions related to the accuracy or integrity of any part of the work are appropriately investigated and resolved.

Open Access Statement: This is an Open Access article distributed in accordance with the Creative Commons Attribution-NonCommercial-NoDerivs 4.0 International License (CC BY-NC-ND 4.0), which permits the noncommercial replication and distribution of the article with the strict proviso that no changes or edits are made and the original work is properly cited (including links to both the formal publication through the relevant DOI and the license). See: https://creativecommons.org/licenses/by-ncnd/4.0/.

\section{References}

1. Der E, Suryawanshi H, Morozov P, et al. Tubular cell and keratinocyte single-cell transcriptomics applied to lupus nephritis reveal type I IFN and fibrosis relevant pathways. Nature immunology 2019;20:915.

2. Zhong S, Zhang S, Fan X, et al. A single-cell RNA-seq survey of the developmental landscape of the human prefrontal cortex. Nature 2018;555:524-8.

3. Aizarani N, Saviano A, Mailly L, et al. A human liver cell atlas reveals heterogeneity and epithelial progenitors. Nature 2019;572:199-204.

4. Ma L, Hernandez MO, Zhao Y, et al. Tumor Cell Biodiversity Drives Microenvironmental Reprogramming in Liver Cancer. Cancer cell 2019:36:418-30.e6.

5. Ramachandran P, Dobie R, Wilson-Kanamori JR, et al. Resolving the fibrotic niche of human liver cirrhosis at single-cell level. Nature 2019:575:512-8.

6. EASL Clinical Practice Guidelines: Liver transplantation. J Hepatol 2016;64:433-85.

7. Bernardi M, Moreau R, Angeli P, et al. Mechanisms of decompensation and organ failure in cirrhosis: From peripheral arterial vasodilation to systemic inflammation hypothesis. J Hepatol 2015;63:1272-84.

8. Iwakiri Y. Endothelial dysfunction in the regulation of cirrhosis and portal hypertension. Liver Int 2012;32:199-213.

9. O'Brien AJ, Fullerton JN, Massey KA, et al. Immunosuppression in acutely decompensated cirrhosis is mediated by prostaglandin E2. Nat Med 2014;20:518-23.

10. Xiong X, Kuang H, Ansari S, et al. Landscape of Intercellular Crosstalk in Healthy and NASH Liver Revealed by Single-Cell Secretome Gene Analysis. Molecular cell 2019;75:644-60.e5.

11. Younossi ZM, Ratziu V, Loomba R, et al. Obeticholic acid for the treatment of non-alcoholic steatohepatitis: interim analysis from a multicentre, randomised, placebocontrolled phase 3 trial. Lancet (London, England) 2019;394:2184-96.

12. Lavine JE, Schwimmer JB, Van Natta ML, et al. Effect of vitamin $\mathrm{E}$ or metformin for treatment of nonalcoholic fatty liver disease in children and adolescents: the TONIC randomized controlled trial. JAMA 2011;305:1659-68.

Cite this article as: Zou ZY, Fan JG. Percutaneous liver biopsy: another option of hepatic sample collection approach in singlecell sequencing studies? Ann Transl Med 2020;8(16):1033. doi: 10.21037/atm.2020.04.23 